\title{
Endothelial cell plasticity at the single-cell level
}

\author{
Alessandra Pasut $^{1,2} \cdot$ Lisa M. Becker ${ }^{1,2} \cdot$ Anne Cuypers $^{1,2} \cdot$ Peter Carmeliet $^{1,2,3,4}$
}

Received: 5 May 2021 / Accepted: 12 May 2021 / Published online: 1 June 2021

(c) The Author(s) 2021

\begin{abstract}
The vascular endothelium is characterized by a remarkable level of plasticity, which is the driving force not only of physiological repair/remodeling of adult tissues but also of pathological angiogenesis. The resulting heterogeneity of endothelial cells (ECs) makes targeting the endothelium challenging, no less because many EC phenotypes are yet to be identified and functionally inventorized. Efforts to map the vasculature at the single-cell level have been instrumental to capture the diversity of EC types and states at a remarkable depth in both normal and pathological states. Here, we discuss new EC subtypes and functions emerging from recent single-cell studies in health and disease. Interestingly, such studies revealed distinct metabolic gene signatures in different EC phenotypes, which deserve further consideration for therapy. We highlight how this metabolic targeting strategy could potentially be used to promote (for tissue repair) or block (in tumor) angiogenesis in a tissue or even vascular bed-specific manner.
\end{abstract}

Keywords Endothelial cells $\cdot$ Single-cell analyses $\cdot$ Angiogenesis $\cdot$ Tissue repair $\cdot$ Tumorigenesis $\cdot$ Metabolism

\section{Introduction}

The vascular endothelium is heterogeneous as it dynamically engages in different functions, influenced by the physiological needs, energetic demands, and distinct conditions of different tissues. These functions include the delivery and exchange of oxygen/nutrients, the regulation of blood flow dynamics (vasoregulation) and hemostasis, the maintenance of tissue-blood barrier functions (trafficking of immune cells, transport of proteins), and tissue-specific angiocrine functions, among others [1-3]. The ability to interrogate

\section{Peter Carmeliet is a lead contact}

Peter Carmeliet

peter.carmeliet@kuleuven.vib.be

1 Laboratory of Angiogenesis and Vascular Metabolism, Vesalius Research Center, VIB, K.U.Leuven, Campus Gasthuisberg, Herestraat 49, B-3000 Leuven, Belgium

2 Laboratory of Angiogenesis and Vascular Metabolism, Department of Oncology, KU Leuven, Leuven, Belgium

3 Laboratory of Angiogenesis and Vascular Heterogeneity, Department of Biomedicine, Aarhus University, 8000 Aarhus C, Denmark

4 State Key Laboratory of Ophthalmology, Zhongshan Ophthalmic Center, Sun Yat-Sen University, Guangzhou, Guangdong, P.R. China the transcriptome at the single-cell level has improved our understanding of EC heterogeneity. Indeed, recent EC-centered single-cell studies revealed a remarkable transcriptional heterogeneity of ECs across vascular beds and tissues, leading to the generation of new concepts and identification of previously unrecognized EC subtypes [4-13].

While we recognize that mural and smooth muscle cells are also important players of the vasculature, we mainly focus here on EC heterogeneity and highlight key examples of EC plasticity, including (i) the diversification/specification of the vascular endothelium in distinct subtypes and (ii) EC plasticity (the ability of quiescent ECs to acquire an angiogenic phenotype). Endothelial-to-mesenchymal transition (EndMT) [14] and the specification of the lymphatic endothelium are other examples of EC plasticity, previously highlighted in recent reviews [14-18].

Focusing primarily on data emerging from single-cell studies, we highlight key mechanisms driving EC plasticity in health and disease, including transcription factor dynamics, epigenetic or tissue-specific cues, and metabolic transcriptome plasticity [19]. We discuss how the vascular endothelium is endowed with different functions that are carried out by specialized EC subtypes, capable of switching phenotypes partially through reprogramming their metabolism. Identification of functionally relevant ECs and the instructive signals that regulate their plasticity will be 
instrumental to develop novel approaches for anti- or proangiogenic therapeutic strategies [20-22]. Here, without an intention to provide an all-encompassing historic overview, we revisit classic and emerging concepts of EC heterogeneity in development and disease, focusing also on recent single-cell data.

\section{Endothelial cell heterogeneity and plasticity}

\section{Diversification of endothelial cells}

The specification of an arterial, capillary, venous, and lymphatic EC phenotype is a critical event for the development of the vasculature [2, 13, 23-25]. Lineage tracing experiments combined with gene reporter activity were among the first to proof the ability of ECs to interconvert between arterial and venous phenotypes. These experiments showed a requirement for VEGF, Notch, and COUP-TF2 in the specification of vessel identity, with higher expression of Notch and VEGF specifying the arterial fate versus COUP-TF2 inhibiting Notch signaling in vein ECs [23, 24, 26-28].

Technological advancements in single-cell -omics studies expanded the opportunities to study EC heterogeneity. To date, single-cell atlases have been constructed from nearly every organ, but only a few of those studies, in particular only when combined with EC enrichment strategies via genetic labeling or use of an EC surface marker, exhibit sufficient power to discover novel EC phenotypes [4-8, 10, 12, 29-31]. A pioneering single-cell transcriptomics study identified the gene expression signature of putative arterial, venous, and capillary ECs in the murine brain, revealing "EC zonation" of these signatures characterized by gradual changes in gene expression [10]. When examining the molecular functions of the most highly expressed genes in each subtype, the authors observed that transcription factors were over-represented in arterial ECs, while transporter expression dominated in capillary and venous ECs [10] (Fig. 1A).

Earlier bulk transcriptomics studies revealed that ECs from different tissues have distinct transcriptomic signatures [32]. These findings have now been expanded by single-cell RNA sequencing studies. In one of the (to date) largest follow-up single-cell RNA sequencing studies using ECs, isolated from 11 adult healthy murine tissues, up to 78 EC populations were identified [4]. Several key insights were deduced from this study [4]. First, ECs from some tissues clustered together and their transcriptomes were more similar to each other, likely because their vasculature shared biological processes (Fig. 1B). For instance, brain and testis ECs resembled each other transcriptomically because they share a tight blood-tissue barrier, while small and large intestinal ECs share a vascular-gut barrier, etc. Second, compared to arterial, venous, and lymphatic ECs, which share multiple common markers across tissues and express few tissue-specific markers (suggesting

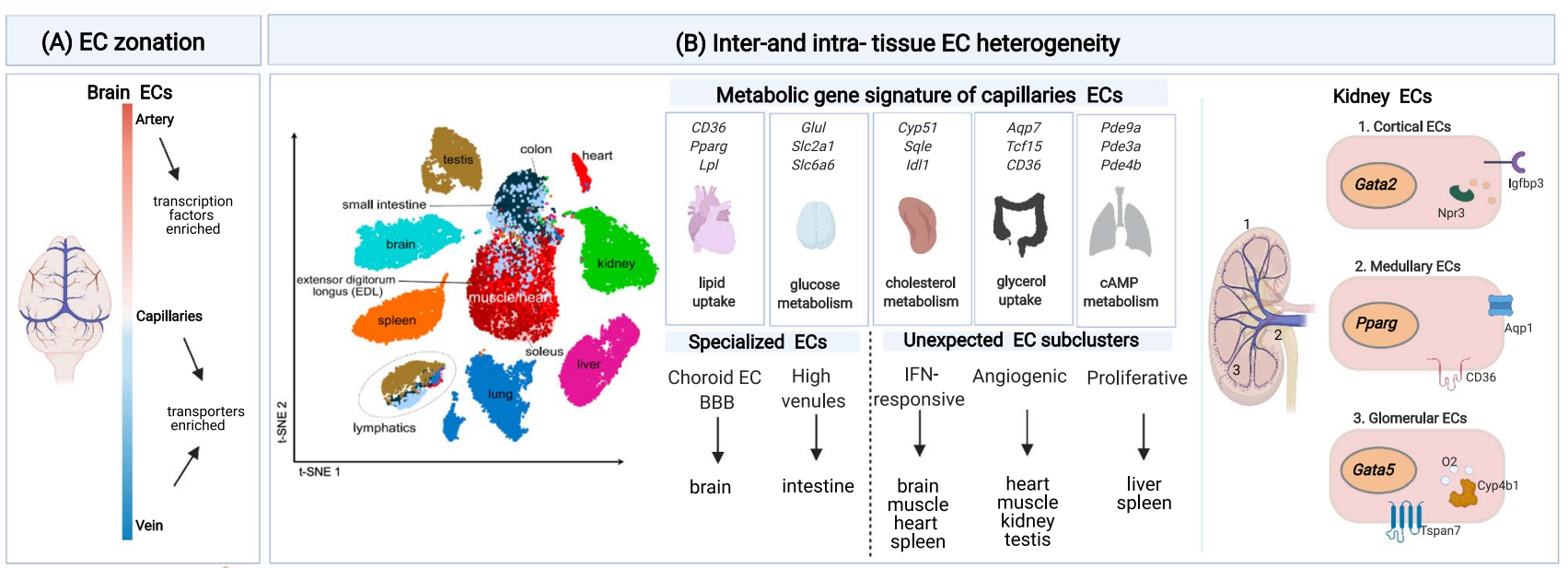

Fig. 1 EC heterogeneity: A Illustration of EC heterogeneity in the brain along the arteriovenous axis (cellular zonation), as unveiled by scRNA-seq adapted from 10. Color-coded bar indicates the gradual changes in gene expression along the arteriovenous axis. Transcription factor genes are enriched in arteries, whereas genes encoding transporters are increased in capillaries and veins. B Single-cell transcriptomic studies of ECs in adult tissues revealed both intra- and inter-tissue heterogeneity. Left: t-SNE plot showing that ECs from some tissues cluster together based on transcriptomic similarities adapted from 4. Middle: Depiction of tissue-specific metabolic gene signatures of capillary ECs, specialized tissue-restricted EC phenotypes (choroid ECs in the brain; high endothelial venule (HEV)-like ECs in the intestine 4), and unexpected EC phenotypes, as identified by scRNA-seq studies. Unexpected EC phenotypes include IFNactivated ECs (found in brain, muscle, heart, and spleen), angiogenic ECs (found in heart, muscle, kidney, and testis), and proliferating ECs (found in liver and spleen). Right: EC heterogeneity in the kidney, determined by topological position, illustrated by some key enriched genes expressed by cortical, medullary, and glomerular ECs. O2: oxygen 
relative transcriptomic stability), capillary ECs express fewer common markers, shared across tissues, and instead are characterized by tissue-specific markers, likely reflecting organ-specific metabolic and physiological needs [4]. In addition to the traditional (artery, capillary, vein, lymphatic) EC phenotypes, tissue-restricted and novel EC phenotypes were identified (proliferating capillaries in liver and spleen; angiogenic ECs in heart, muscle, kidney, and testis; IFNactivated ECs in brain, muscle, heart, and spleen) (Fig. 1B) $[4,6]$. Third, ECs in vascular beds (arteries, capillaries, veins) transcriptomically resemble each other across tissues $[4,20]$. Finally, not the position in the vascular tree, but the tissue type primarily contributes to EC heterogeneity [4, 9-11] (Fig. 1B).

Other analyses, such as re-analysis of single-cell RNA sequencing of ECs from the Tabula Muris consortium [8] and microarray analyses, reported similar findings of tissuespecific gene expression in ECs [8]. Pathway analysis of the most differentially expressed genes (DEG) in ECs across tissues showed that while some pathways are common to all ECs irrespective of the tissue of origin, tissue-specific preferences dictate which particular set of genes is expressed in ECs in any given tissue $[4,20]$, further highlighting tissue-specific EC specialization. An example is represented by brain ECs, which are heterogeneous (with EC subtypes unique to the brain, such as the choroid plexus ECs) compared to ECs from other tissues (heart and muscle) [4, 10, 20, 32]. In addition, even within the same organ, ECs are transcriptionally heterogenous depending on their topological (compartment-specific) position. For example, in the kidney, cortical, medullary, and glomerular ECs are transcriptionally distinct. Cortical ECs express high levels of Ifg bp 3 and $\mathrm{Npr} 3$ and the transcription factor Gata2, whereas medullary ECs are enriched for Pparg and the fatty acid transporter CD36 and Aqp I (the latter can only be found in a subset of medullary ECs). Lastly, glomerular ECs upregulate the expression of Gata5, Tspan7, and Cyp4b1 (among other genes) (Fig. 1B). Within the same compartment, distinct subtypes of ECs were identified including capillary renal ECs with an angiogenic signature and renal vein and capillary ECs subtypes with an IFN signature [9]. The microvasculature of the liver also represents an additional example of tissue zonation, recently discussed elsewhere [11].

The opportunity to combine lineage tracing strategies with single-cell transcriptomic approaches provides an opportunity to (re)-investigate developmental pathways and to generate novel mechanistic insights by inferring the developmental history of single cells based on their transcriptional profile [12, 33, 34]. One such example is the reinvestigation at single-cell level of coronary artery specification during embryonic development [12]. An accepted view was that blood flow dynamics shape the development of the coronary vasculature $[27,35,36]$. Reconstruction of developmental trajectories of single ECs in the sinus venous using single-cell RNA sequencing showed the existence of a rare type of ECs in veins in the developing murine heart (at E12.5) that can adopt a pre-arterial fate (independently of blood flow dynamics) and eventually differentiates into coronary arterial ECs [12] (Fig. 2A). Of note, in this subset of cells, the expression of CoupTFII was decreased, allowing the differentiation of pre-artery cells into arteries. Overexpression of CoupTFII blocked the specification of pre-artery cells and their differentiation into arteries (via upregulation of cell cycle genes). This new finding not only adds to our understanding of how artery and vein ECs develop and are inherently plastic, but also may have implications for the treatment of heart disease and the promotion of arterialization [21]. Interestingly, gene fate mapping combined with clonal analysis showed that a subset of embryonic endocardial ECs transition into $P d g f r \alpha / \beta$-expressing progenitors, which eventually convert into pericytes and vascular smooth muscle cells (vSMCs) in the heart [37] (Fig. 2B).

\section{Differentiation of endothelial cells to other cell types}

Adult ECs can be reprogrammed to differentiate to competent hematopoietic stem cells (HSCs), a process driven by overexpression of the transcription factors Fosb, Gfil, Runxl, and Spil [38]. Inductive signals from the vascular niche also contribute to the reprogramming [38]. Indeed, genetically reprogrammed ECs co-cultured in the presence of "vascular niche" cells showed increased self-renewing capacity [38] (Fig. 2B). "Vascular niche" cells were ECs overexpressing E4ORF1, which have been shown to maintain an angiogenic phenotype and are capable of HSC maintenance and expansion (Fig. 2B). Inductive angiocrine signals, including CXCL12 and BMP, sustained the expansion and self-renewal of the reprogrammed EC clones. Analysis of single-cell trajectories of the endothelial-to-hematopoietic transition from human pluripotent stem cells (hPSCs) similarly showed that ECs are capable of generating hematopoietic cells in a process governed by RUNX1, TAL1, and GATA2 [39]. Here, regulation of cell cycle dynamics appeared critical for the reprogramming into the hematopoietic lineage [39] (Fig. 2B).

In addition to the endothelial/hematopoietic axis, ECs bear the potential to differentiate to cardiac and skeletal muscle cells. Etv2 is a transcription factor expressed by vascular progenitors (including "embryonic ECs"), important for the regulation of the endothelial/ hematopoietic axis [40-42] (Fig. 2B). A single-cell RNA sequencing study of zebrafish embryos showed that loss of Etv2 precluded the differentiation of endothelial and myeloid cells, while instead ECs acquired alternative fates including cardiomyocytes and a somitic fate via upregulation of myogenic 


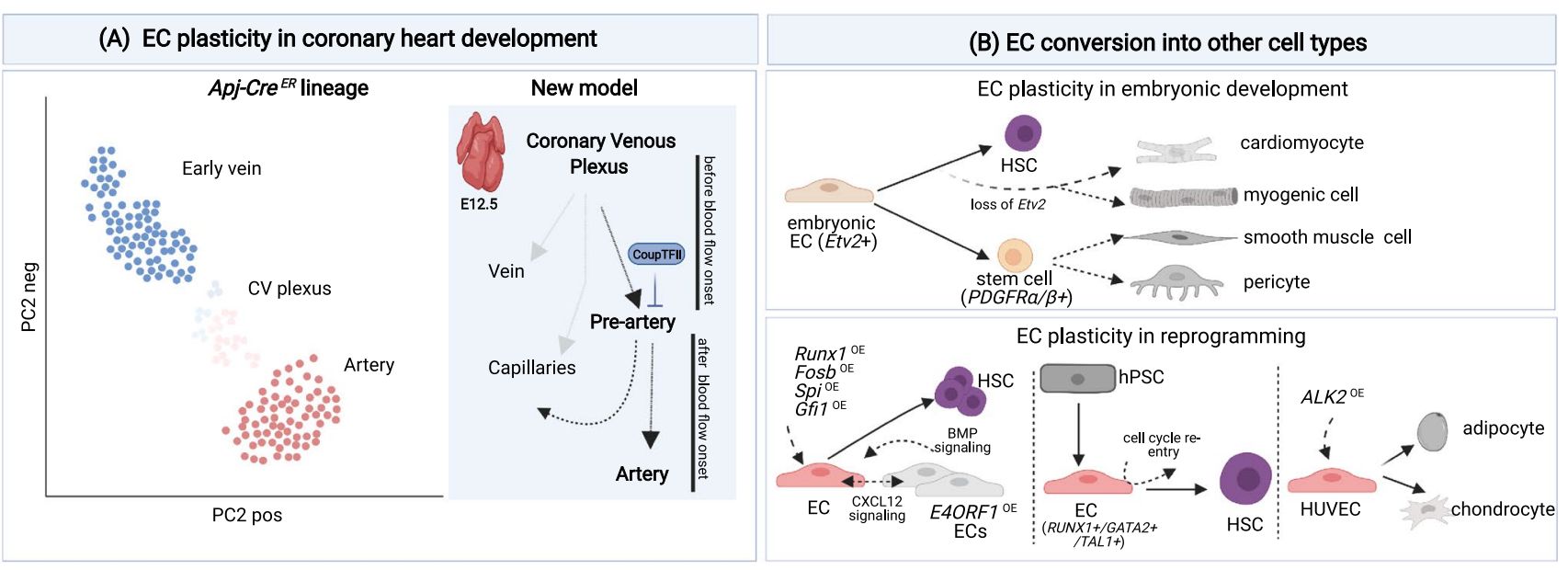

Fig. 2 EC plasticity: A Randomized principle component analysis (rPCA) plot of single ECs in the sinus venous from Apj-Cre mice intercrossed with $\operatorname{Rosa}^{m T m G}$ reporter mice (to trace cells from the sinus venous), revealed clusters of early vein, coronary venous (CV), and artery ECs adapted from 12. The model proposes that during embryonic development (E12.5), ECs in the coronary venous plexus can adopt a pre-arterial fate before the onset of blood flow and eventually differentiate into coronary arterial ECs. CoupTFII inhibits prearterial to arterial EC differentiation. A subset of pre-arterial ECs differentiates into capillaries, while a fraction of ECs from the coronary venous plexus is also capable of differentiating into capillaries. B Top panel: Examples of EC plasticity during development. Embryonic ECs (expressing Etv2; key regulator of the endothelial/hematopoietic axis) are capable of differentiating into cardiac and skeletal muscle (myogenic) cells when Etv2 expression is silenced. In the presence of Etv2, embryonic ECs can differentiate into hematopoietic stem

markers [40] (Fig. 2B). In developing chicken limbs, ECs and somites share a common developmental origin [43], and in the mouse, somite Notch signaling promotes a vascular cell-fate choice at the expense of a myogenic fate [44]. In addition, overexpression of a constitutively active form of ALK2 is sufficient to promote the differentiation of ECs into adipocytes and chondrocytes [45] (Fig. 2B). Thus, ECs can differentiate to other cell types. Whether such plasticity can be exploited for therapeutic strategies (cell therapy, generation of blood vessel organoids, treatment of malignancies and immunological disorders, etc.) remains to be determined (see Box Exploitation of EC plasticity for regenerative medicine) $[46,47]$.

\section{Angiogenic EC phenotypes}

\section{New vessel formation}

During embryonic development, numerous vessels are formed from pre-existing ones, in a process called angiogenesis, which can be further divided into sprouting angiogenesis (SA) and intussusceptive angiogenesis (IA) [1, 25, cells (HSCs). Embryonic ECs can also transition into stem-like cells (Pdgfr $\alpha / \beta+$ progenitors) that can further differentiate into mural cells (pericytes and smooth muscle cells) in the heart. Bottom panel: Examples of EC plasticity in different settings of reprogramming. Left, Adult ECs can be reprogrammed by transcription factor overexpression (Fosb, Gfil, Runxl, Spil) to differentiate into competent hematopoietic stem cells. ECs overexpressing E4ORF1 (presumed surrogates of niche cells) contribute to HSC maintenance and expansion via angiocrine CXCL12 and BMP signaling. Middle, human pluripotent stem cell (hPSC)-derived ECs expressing RUNX1, TAL1, and GATA2 can differentiate to HSCs. The full differentiation requires cell cycle re-entry. Right, Overexpression of a constitutively active form of the activin receptor-like kinase-2 (ALK2) converts HUVECs to adipocytes and chondrocytes. CV: coronary venous, HSC: hematopoietic stem cell, OE: overexpression, HUVECs: human umbilical vein endothelial cells

48]. SA regulates the growth of vessels via sprouting of ECs. In contrast, during IA, pre-existing vessels split and ECs extend inside the vessel and build a new lumen within the pre-existing vessel (via formation of tissue pillars) [49]. This angiogenic mechanism not only allows to grow new vessels via duplication (intussusceptive microvascular growth) but also to remodel the vascular tree via arborization (intussusceptive arborization) and pruning (intussusceptive pruning), contributing to the control of the vascular tree geometry [49]. Angiogenesis in healthy adult organs is rare, with a few exceptions (skeletal muscle angiogenesis during physical exercise; endometrial angiogenesis during epithelial regeneration $[50,51])$. However, upon injury, adult ECs can rapidly grow new vessels via re-activation of developmental angiogenic programs (SA or IA).

During retinal vascularization, a model of SA has been put forward [52-56]. Angiogenic signals (such as VEGF) induce the formation of a "tip" cell with long filopodia that sense these signals. The tip cell then guides the nascent vessel sprout to the site of VEGF production and at the same time also upregulates the expression of its receptor (Vegfr2) (Fig. 3A) [53, 54]. Following the tip cell, proliferating "stalk" cells elongate the sprout (Fig. 3A). When the 
(A) Traditional angiogenic ECs

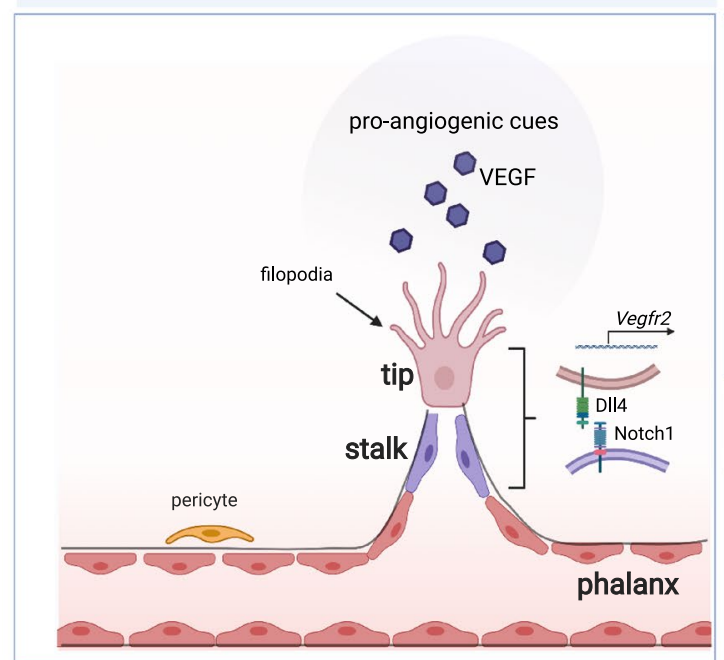

(C) rESCs with regenerative potential

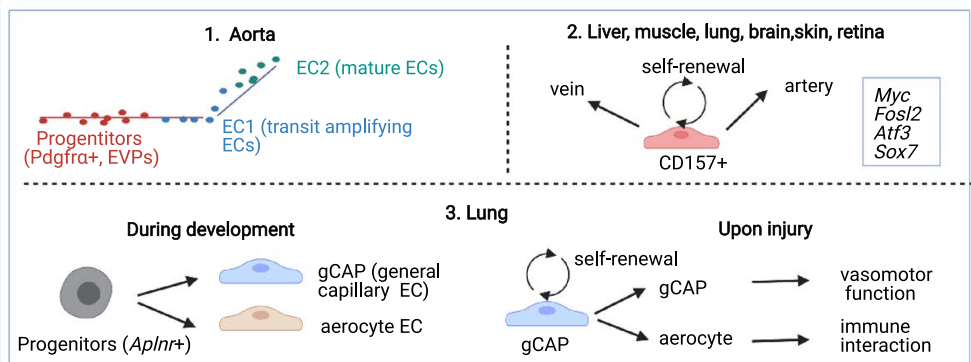

(D) Alternative modes of vessel repair

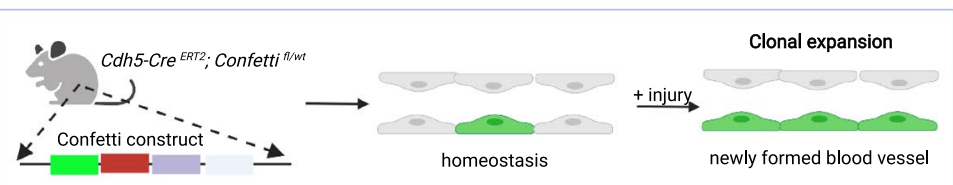

(B) New angiogenic phenotypes

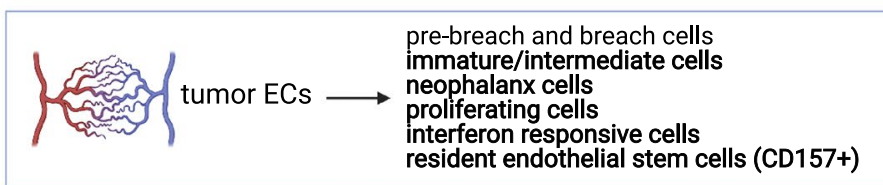

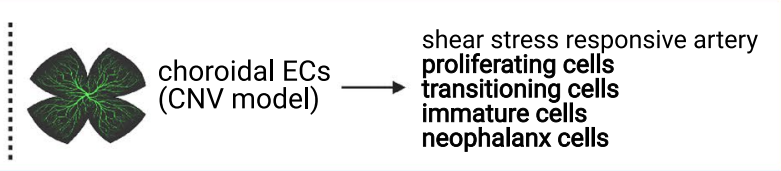

Fig. 3 Mechanisms of vessel repair: A Illustration of SA, showing the leading tip EC (with filopodia) and stalk ECs and some key molecular regulators of the tip/stalk specification via the VEGF/Notch axis upon pro-angiogenic cues. Tip cells are characterized by high expression of Dll4 and VEGFR2 (receptor for VEGF). Dll4 signaling from the tip cells induces the expression of Notch signaling in stalk cells. Quiescent phalanx ECs are indicated. B List of EC phenotypes identified by scRNA-seq in models of pathological angiogenesis (tumors; choroidal neovascularization (CNV)). Conserved EC phenotypes across models of CNV and lung cancer $[6,31]$ are shown in bold. EC phenotypes identified in only one of the two models (tumor or CNV) include breach and pre-breach ECs (tumor-restricted) and shear stress responsive arterial ECs (only in CNV). C Scheme of resident endothelial stem cells (rESCs) endowed with regenerative potential: 1. Pseudotime analysis of aortic ECs showing the differentiation trajectories of $P d g f r \alpha+$ endovascular progenitor cells (EVPs) (red line) into two distinct EC subtypes, i.e., EC1 (green top line; transit ampli-

new vessel becomes perfused, ECs differentiate to quiescent "phalanx" cells. Notably, stalk cells can dynamically differentiate to tip cells and overtake the latter, so that the fittest EC leads the vessel sprout at the forefront [53, 55-57]. Hence, tip and stalk cell phenotypes are not genetically predetermined, fixed states, but instead represent plastic EC phenotypes (Figs. 3A and 4A).

Single-cell studies of choroidal neovascularization (CNV) and cancer expanded our view on the phenotypic heterogeneity of SA and identified several EC clusters [6,31]. These include typical angiogenic EC phenotypes (tip and proliferating ECs) and more mature phalanx ECs upon formation of more mature vessels; curiously, typical bona fide stalk ECs fying ECs) and EC2 (blue bottom line; mature ECs) adapted from 66. 2. CD157+rESCs (resident endothelial stem cells, capable of selfrenewal and differentiation to artery and vein ECs) are found in large vessels of several organs (liver, muscle, lung, brain, skin, and retina). Key transcription factors expressed by CD157+ECs are listed in the box on the right. 3. Aplnr + progenitor cells in the lungs differentiate into general capillary (gCAP) ECs and aerocyte ECs during embryonic development. Upon lung injury in adult tissues, gCAP ECs are capable of self-renewal and differentiation into aerocytes. gCAP ECs are important in vasomotor control, whereas aerocytes are involved in immune functions (leukocyte trafficking). D Clonal expansion represents an alternative mode of vessel repair. Genetic tracing can be used to identify clonal expansion of ECs during vessel repair. Here, mice harboring the Confetti marker were crossed to Cadherin5-Cre ERT2 animals to trace clonal expansion of Cadherin5+ ECs upon injury [109]

were not detected, though EC phenotypes expressing some stalk cell features were identified [6, 31]. Newly identified EC subtypes included immature and neo-phalanx ECs [31], and breach cells, i.e., an EC subpopulation sharing gene markers with tip cells, but also expressing genes involved in podosome rosette formation to prepare for/assist tip cell migration [6] (Fig. 3B). By contrast, to date, nearly nothing is known about the molecular and cellular heterogeneity of ECs during IA.

Recent single EC transcriptomic studies using pseudotime analysis enabled in silico lineage tracing of ECs during SA. These analyses revealed that, in neovascular diseases, post-capillary venule ECs initiate vessel sprouting 


\section{(A) EC metabolism in blood vessel growth}

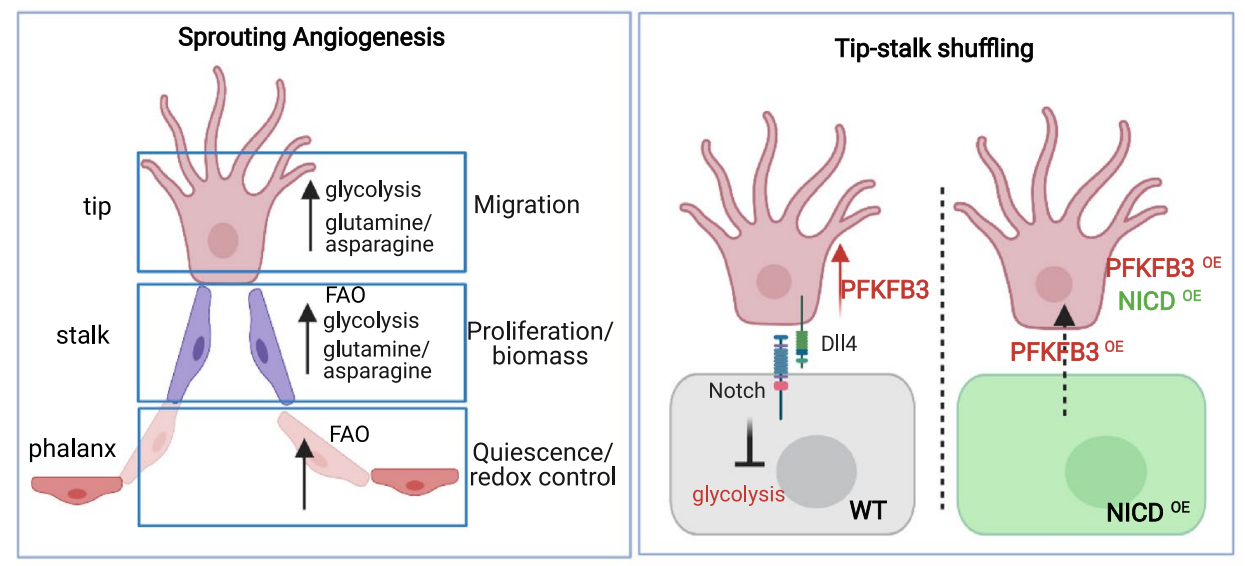

(B) Proposed EC-based therapeutic strategies

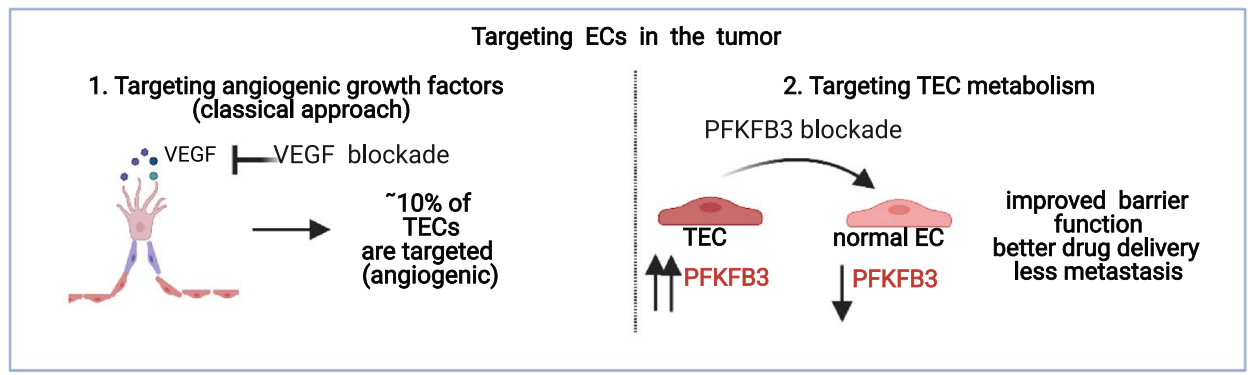

Fig. 4 EC metabolism and proposed new EC-targeted therapies: A EC metabolism during sprouting angiogenesis: Left: Key metabolic pathways associated with each EC phenotype (tip, stalk, and phalanx ECs) involved in sprouting angiogenesis. Glycolysis and amino acid metabolism (glutamine/asparagine) are increased in tip cells to support migration. Fatty acid oxidation (FAO), glycolysis, and amino acid metabolism (glutamine/asparagine) support biomass production in stalk cells, while in phalanx ECs high levels of FAO support the maintenance of the quiescence phenotype (partially via regulation of the redox balance). Right: Regulation of tip-stalk cell shuffling: the glycolytic activator PFKFB3 is upregulated in tip cells while Notch signaling inhibits glycolysis in stalk cells. Overexpression of the Notch intracellular domain (NICD) maintains high Notch signaling and the stalk cell phenotype. However, PFKFB3 overexpres- sion is sufficient to turn a stalk cell into a tip cell, even in the case of parallel overexpression of NICD. Therefore, PFKFB3 signaling overrules Notch signaling and drives the tip cell phenotype, and metabolism can fuel vessel sprouting independently of angiogenic signals. B Proposed EC-based therapeutic targeting strategies in cancer: 1 . Fewer than $10 \%$ of tumor ECs are targeted by classic antiangiogenic therapy (anti-VEGF), since such therapies primarily target angiogenic ECs and not the majority of other tumor ECs, which may partly explain therapy resistance. 2 . Targeting of EC metabolism has emerged as a promising approach to target (tumor) angiogenesis. Inhibition of glycolysis via PFKFB3 blockade in tumors resulted in enhanced barrier function, decreased metastasis, and improved drug delivery in animal models and then progress over intermediate states to differentiate to tip cells and subsequently to more quiescent phalanxlike ECs, once vessel formation has been established [31]. Of note, cells expressing genes associated with resident endothelial stem cells (rESCs) were enriched in post-capillary venules in tumors [6] (Fig. 3B), raising the question whether they might contribute to vessel formation and regeneration, as shown previously [58, 59]. Notably, since the venous EC population (containing rESCs) expanded upon anti-VEGF treatment of tumor-bearing mice, these findings raise the question whether an enlarged pool of
rESCs may contribute to the resistance against anti-VEGF therapy.

During development, Notch and VEGF regulate arterial fate and vessel sprouting [60, 61]. In SA, VEGF induces the migration of tip cells and the expression of D114. D114 activates Notch in stalk cells, impairing acquisition of the tip phenotype [53, 54] (Fig. 3A). Depending on the level of Notch expression, ECs may sprout or differentiate to arteries while exiting the cell cycle. On the contrary, COUP-TF2 inhibits the arterial fate and at the same time upregulates cell cycle genes [23, 26, 62]. Of note, the interplay between 
cell-fate decisions, angiogenesis, and cell cycle has been re-investigated. Using a combination of bulk transcriptomic and fate mapping approaches in mosaic mice with heterogeneous Notch expression [28], Notch, rather than directly regulating the expression of arterial fate genes, inhibits the transcriptional activity of Myc and downstream cell cycle and metabolic genes, suggesting a role for cell cycle and metabolic genes in the regulation of SA and EC identity [28].

\section{Vessel repair}

ECs are continuously exposed to circulating pathogens, toxic substances, and hazardous agents and therefore can undergo damage, requiring continous repair/regeneration. Also, diseases such as diabetes, atherosclerosis, and others are characterized by EC dysfunction [63]. Notably, single-cell RNA sequencing of ECs revealed a small number of angiogenic ECs in healthy quiescent tissues [4], raising the question whether they might be involved in new vessel formation or regeneration of dysfunctional ECs. In agreement, analysis of the EC renewal rate in the heart of individuals between 20 and 70 years of age revealed several cycles of EC turnover throughout adulthood [59, 64].

With regard to the mechanisms of vascular repair, a matter of debate is whether different EC subtypes with stem-like features exist in all or only in large vessels and if additional (non-EC) cells may participate in this process [58, 65-70]. Two single-cell studies identified possible novel mechanisms of EC regeneration [58, 65]. Both studies showed that vascular regeneration is largely driven by vessel wall-resident ECs, therefore excluding (at least in the used model systems) a major contribution by circulating stem or hematopoietic progenitors [69-72]. In one study, regeneration of the endothelium relied on a relatively rare population of CD31 +/CD45-rESCs, enriched for the expression of the surface markers CD157 and CD200 and for several transcription factors including Myc, Fosl2, Atf3, and Sox 7 and detected in large vessels of several organs (liver, heart, lung, intestine, skin, muscle, and brain) [65] (Fig. 3C). In two different models of tissue repair (liver regeneration, hindlimb ischemia), CD157+ECs were capable of repairing the vessel via activation of an angiogenic program [65]. In a second report, repair of the aorta was driven by ECs and not by circulating progenitors [58]. ECs close to the site of injury were responsible for regeneration of the damaged vessel and the gene expression profile of these regenerating ECs did not resemble that of sprouting ECs [58]. In fact, these regenerating ECs show a gene signature indicative of a coordinated and rapid switch from quiescence to highly proliferative cells with genes associated with maintenance of cell junctional complexes. Moreover, genes associated with migratory behavior were absent in these cells. Also, single-cell analysis identified quiescent endovascular progenitors (EVPs) in the aorta, characterized by a high mitochondrial content (as observed in other stem cells/progenitors) $[66,73,74]$, enriched in mesenchymal markers (such as PDGFR- $\alpha$ ), which transitioned to two differentiated EC populations (transit amplifying ECs and mature ECs) upon pseudotime trajectory analysis [66] (Fig. 3C). Thus, vessel wall-resident EC progenitors may contribute to repair of damaged ECs.

In addition to large vessels, the microvasculature (in lungs) may also harbor stem/progenitors for ECs [67]. During embryonic development, Aplnr + progenitor cells in the lungs differentiate into general capillary (gCAP) ECs, which are responsible for vasomotor control and aerocytes (lungspecific ECs important for leukocyte trafficking). In adult tissues, gCAP ECs themselves were capable of self-renewal and differentiation into aerocyte ECs during lung injury, demonstrating their stem/progenitor-like features $[67,75]$ (Fig. 3C). Similarly, a subpopulation of capillary ECs with stem-like features exists in the microvasculature of lymphoid tissues [68].

\section{EC heterogeneity in cancer}

Angiogenesis is a hallmark of cancer. Earlier studies using bulk or targeted approaches revealed that tumor ECs (TECs) differ from their normal counterparts and exhibit considerable heterogeneity [22, 76-79]. For instance, single-cell RNA sequencing and comparative microarray analysis revealed that TECs from human lymphoma, breast, and colorectal cancer display a distinct gene expression signature compared to their healthy counterparts, enriched in genes involved in extracellular matrix (ECM) metabolism and collagen formation, vascular smooth muscle contraction, and signaling pathways [80].

Recent single-cell studies expanded these insights considerably. Single-cell analysis of human non-small cell lung cancer (NSCLC) discovered distinct transcriptome signatures in TECs compared to ECs from non-malignant lung tissue [81], with Myc targets presenting the top upregulated pathway in TECs, in line with Myc's role in tumor angiogenesis [82]. Surprisingly, genes involved in antigen presentation (MHC class I and II), chemotaxis (CCL2, CCL18, ILO), and immune cell homing (ICAMI) were downregulated in TECs compared to normal lung ECs, raising the question whether TECs potentially contribute to immune tolerance [81]. In agreement, compared to early-stage ground glass nodules adenocarcinoma (GGN-ADC), TECs from latestage solid adenocarcinoma (SADC) exhibited lower levels of genes involved in antigen presentation and chemotaxis [83]. 
Follow-up single-cell RNA sequencing of isolated ECs from paired human NSCLC and peri-tumoral tissue and from murine lung tumors and healthy lungs provided additional understanding [6]. These studies together identified 33 EC phenotypes in tumor and healthy lung tissue and yielded the following insights [6]. First, fewer than $10 \%$ of TECs in human NSCLC were angiogenic tip or proliferating ECs, raising the question whether this contributes to the resistance of VEGF-blockade anti-angiogenic therapy and can explain its relative insufficient efficacy [6] (Fig. 4B). Traditional stalk cells were not detected [6]. Second, capillary ECs in healthy lung expressed a gene signature of semi-professional antigen-presenting cells, but these immunomodulatory ECs are underrepresented in lung tumors [6]. In agreement, genes related to antigen presentation and chemotaxis were downregulated in TECs from SADC compared to early-stage GGN-ADC [83]. On the other hand, ECs with transcriptomic features of high endothelial venules (HEVs) were more abundant in tumors [84], suggesting a complex regulation of tumor immunity by TECs. Third, breach TECs were identified, likely assisting tip cell migration (Fig. 3B, see above) [6]. Fourth, upon treatment of tumor-bearing mice with VEGF-targeted compounds, tip and breach TECs were most sensitive, proliferating TECs were not more sensitive (likely indicating that other angiogenic signals were driving TEC proliferation), while venous TECs were less sensitive [6]. In agreement, venous TECs expanded upon VEGFblockade therapy. Given that venous ECs contained rESCs (see above), it remains to be determined whether any possible expansion of this population might contribute to the resistance against VEGF-blockade therapy [6].

Generating long lists of gene markers for distinct cell populations by single-cell RNA sequencing (scRNA-seq) is becoming a standard procedure; however, utilizing this data tsunami to identify and prioritize new angiogenic targets is a formidable challenge. One study hypothesized that EC phenotypes or gene markers, congruently upregulated across species, diseases, tissues, and experimental conditions, would represent biologically more important candidates and therapeutically more attractive targets [6]. An integrated multi-disciplinary approach, involving scRNAseq, complemented with validation bulk transcriptomics and proteomics studies, illustrated the potential of this approach for identifying new angiogenic targets, which were functionally validated in vitro and in vivo [6] (Box Novel integrated analysis to identify \& validate scRNA-seq targets).

A limitation of current research on EC heterogeneity in cancer is that still very little is known about alternative modes of tumor vessel formation, including vessel splitting (IA) [85, 86] and vessel co-option [87]. Nonetheless, tumors may switch from SA to vessel splitting or co-option when treated with VEGF blockade [85, 88, 89]. These alternative tumor vascularization modes may thus help escape the tumor from and cause resistance to traditional anti-angiogenic therapy.

\section{EC heterogeneity in tissue repair}

In 2019, a new strain of Coronavirus, severe acute respiratory syndrome (SARS)-CoV-2, caused a global pandemic. COVID-19, the disease caused by SARS-CoV-2 infection, is characterized in severe cases by life-threatening acute respiratory distress syndrome (ARDS) in the lungs and by widespread multi-organ failure [90]. A perspective hypothesized that EC dysfunction contributed to COVID-19 vascular complications (vascular leakage, thrombosis, inflammation) [90-95]. Also, vessel splitting (IA) appeared to be more prevalent in COVID-19 lungs [92, 93]. Even though neovascularization is a common denominator of other lung diseases (acute lung injury [96], idiopathic pulmonary fibrosis [97]), it remains elusive which particular EC subtypes are involved. We will highlight a few examples of the role of ECs in tissue repair and alternative EC functions, which have recently become more evident.

First, ECs may modulate immune responses as "immunomodulatory ECs (IMECs)" [4, 98, 99]. Analysis of ribosome-bound mRNAs (reflecting functionally relevant transcriptome changes) of ECs in three different tissues (lung, heart, brain) revealed that inflammation (induced by LPS) resets ECs with a broad representation of genes involved in leukocyte/immune cell trafficking [99]. Notably, LPS-treated ECs from different tissues transcriptomically resembled each other more closely than untreated ECs [99]. In single-cell studies, EC subsets have been identified expressing genes involved in scavenging, immunoregulation, immune surveillance, and interferon signaling across different tissues [4, 8] (Fig. 3B). Identification of markers to discriminate these subtypes coupled with functional studies will be important to address which exact immunomodulatory function (proversus anti-inflammatory) these EC subsets have.

Of note, scRNA-seq and ATAC-sequencing (to characterize the epigenomic landscape) of ECs from mouse carotid arteries after partial ligation illustrated that disturbed flow induced reprogramming of ECs toward an immune cell-like phenotype (coined "EndICLT"), expressing macrophage markers [100]. Tissue-specific endothelial genes (enriched in the brain, heart, lungs, and kidney [4]) were expressed at low or undetectable level in carotid ECs [100], further illustrating the tissue- and vascular bed-specific EC heterogeneity. Notably, disturbed flow also induced this EndICLT phenotype in cultured human aortic ECs, in the absence of immune cells [100]. Thus, ECs are highly plastic cells that transition to immunomodulatory cell phenotypes, identifiable by single-cell analysis as a separate subset [100]. ECs have been previously involved in immune responses [101, 
102]. Such phenotypic plasticity of ECs to IMECs is not unique to ECs, since epithelial cell-to-immunomodulatory cell transition has been reported [103].

Multi-omic approaches (epi-genomic atlases; spatially resolved gene expression) allow to generate in-depth mechanistic insights [104, 105]. In one such study, simultaneous bulk analysis of the transcriptome and epigenome of ECs across 12 organs showed that ECs are epigenetically preprogrammed for a response to immunological challenges, including viral infection [106]. Future studies will inform on the contributions of distinct IMEC subsets to immunity in normal tissue homeostasis, tumor immunity, and graft rejection reactions [107, 108].

Second, vascular repair in a model of myocardial infarction does not need to rely on SA alone, but can also occur via alternative vascularization modes $[109,110]$. For instance, use of the Confetti reporter mouse line crossed with $\mathrm{Cad}$ herin 5-Cre mice (to enable clonal analysis of Cadherin5+ ECs) revealed that vessel repair can proceed via clonal expansion of proliferative clusters of ECs as determined by the analysis of the fluorescence color distribution of the labeled cells after myocardial injury [109] (Fig. 3D). Such proliferative EC subtypes (characterized by enriched expression of cell cycle and metabolic genes) have also been described in a model of choroidal SA [31]. In addition to SA (which requires EC proliferation), IA represents an alternative mode of vascularization for tissue repair. Indeed, for instance in the lung, SA is detected in usual interstitial pneumonia (UIP) [111] and H1N1-infected lung explants [92], while IA is documented in rat models of glomerulonephritis [112] and colitis [113], in lungs of patients affected by nonspecific interstitial pneumonia (NSIP), alveolar fibroelastosis (AFE) [111], and COVID-19 disease [92].

Third, vascular repair may involve the re-appearance of a known EC subtype in a different location. For instance, in healthy lungs, peri-bronchial ECs are restricted to the bronchial vasculature in large airways, while in idiopathic pulmonary fibrosis (IPF), peri-bronchial ECs (characterized by high COL15Al expression) were enriched in IPF lungs, in particular in areas of bronchiolization and fibrosis [114]. This is likely a compensatory mechanism, whereby destruction of peripheral pulmonary capillaries leads to an increase in pulmonary pressure with hypertrophy and neoangiogenesis of peribronchial vessels.

\section{The role of metabolism in EC plasticity and heterogeneity}

When angiogenic signals stimulate vessel formation, ECs must adapt their metabolism in order to produce the energy and biomass and to secure redox homeostasis, necessary for ECs to divide, grow, and migrate during vessel formation
$[63,115]$. A key transgenic experiment provided the first proof-of-evidence for the essentiality of EC metabolism, illustrating that EC metabolism could even overrule the activity of angiogenic signals [116] (Fig. 4A). As explained above, tip and stalk cells are interchangeable EC phenotypes, with stalk cells overtaking the leading tip position in a highly dynamic process regulated by Notch (high Notch signaling promotes the stalk cell phenotype) [54, 55]. Remarkably, in vivo evidence from a zebrafish SA model showed that over-expression of the glycolytic activator PFKFB3 is sufficient to convert a stalk cell into a tip cell, despite high Notch signaling [induced by the overexpression of the Notch intracellular domain (NICD)], thus overruling Notch-induced stalk cell conversion [116] (Fig. 4A). Conversely, in an in vitro SA model using mosaic EC spheroids, $P F K F B 3$ gene silencing impaired the tip cell competitiveness [115-117]. Of note, low levels of glycolysis in stalk cells contribute to proliferation in these cells (Fig. 4A).

Notably, different metabolic enzymes are capable of driving EC plasticity. Just to give a few examples, carnitine palmitoyl-transferase 1A (CTP1A), a rate-controlling enzyme of fatty acid oxidation (FAO), results in the production of acetyl-CoA to sustain (in conjunction with anaplerotic substrates) the tricarboxylic acid (TCA) cycle to stimulate nucleotide synthesis in proliferating ECs [118], but this enzyme also maintains redox homeostasis in quiescent ECs [119]. Thus, FAO is critical for biomass production in stalk ECs and for quiescence in phalanx ECs during angiogenesis (Fig. 4A). At the same time, FAO promotes venous-to-lymphatic EC differentiation by increasing histone acetylation at lymphangiogenic genes, thereby increasing the expression of the lymphatic master transcription factor Prox-1 [120, 121]. Other metabolic pathways fuel the differentiation of tip cells and stalk cells. For instance, glutamine and asparagine metabolism is essential for stalk-to-tip conversion during SA [122] (Fig. 4A), while fatty acids and ketone bodies promote EC proliferation in blood vessels and lymphatics [123, 124]. When differentiating to ECs, stem/ progenitor cells in the mouse aorta increase glycolysis and oxidative metabolism, a mechanism possibly regulated by AKT/ mTOR signaling [125]. In addition, FOXO1 regulates EC quiescence by suppressing Myc and cellular metabolism [126], while the pro-quiescence transcription factor KLF2 suppresses glycolysis in ECs [127]. Fatty acid synthase (FASN), a critical enzyme in lipid synthesis, proved important for EC proliferation and vessel sprouting through malonylation of mTOR [128]. Another enzyme affecting EC function is the M2 isoform of pyruvate kinase (PKM2), which is important for proliferating ECs to ensure cell cycle progression (via inhibition of NF- $\mathrm{kB} / \mathrm{p} 53$ signaling) [129, 130] and in quiescent ECs to maintain vascular barrier function. Intriguingly, PKM2 has also been involved in modulating EC immune functions via epigenetic control (indirectly 
by impacting S-adenosylmethionine synthesis) of anti-viral innate immune signaling of ECs [129]. For a more detailed description of metabolic regulation of EC biology, we refer to comprehensive reviews $[19,121,131]$.

Single-cell studies revealed a greater metabolic transcriptome heterogeneity than expected. Indeed, when focusing only on the expression of metabolic genes by ECs from different healthy mouse tissues, each EC subtype in each different tissue could be distinguished by a particular metabolic gene signature [4] (Fig. 1B). This was also the case for ECs in diseases, characterized by new vessel formation (tumors in the lung, CNV in the eye) [6, 31]. Notably, ECs from some tissues expressed largely similar metabolic transcriptomes, presumably because they share biological features (see above), suggesting an important contribution of the metabolic transcriptome to the EC identity $[4,31]$. In addition, ECs from different tissues exhibited metabolic transcriptome heterogeneity. For example, brain ECs have a higher expression of glucose and amino acid transporters (Glul, Slc2al, Slc6a6), while splenic ECs show gene expression related to cholesterol metabolism (Cyp51, Sqle, Idll) [4, 19]. In turn, cAMP metabolic gene expression is elevated in lung ECs (Pde9a, Pde3a, Pde3b), while cardiac and muscle ECs exhibit enrichment of genes involved in lipid uptake and metabolism (CD36, Pparg, Lpl) [4, 132] (Fig. 1B). Even more, ECs display metabolic gene signature heterogeneity within the vascular tree, in a tissue type-specific pattern. Indeed, in the liver, ECs from each vascular bed (arteries, capillaries, veins) express distinct metabolic gene markers, while, in the brain, ECs from these vascular beds share common markers or express vascular bed-specific markers [4]. In agreement, ECs from certain vascular beds in the brain showed heterogeneity in the expression of transporter genes [10, 99, 133]. Renal ECs also exhibit zone-dependent metabolic transcriptome heterogeneity. Indeed, compared to glomerular and cortical ECs, medullary ECs were enriched in genes involved in oxidative phosphorylation (OXPHOS), a finding validated by metabolic flux analysis [9]. OXPHOS was essential for medullary ECs to cope with dehydration stress [9].

Compared to normal ECs, proliferating ECs in murine lung tumors and in $\mathrm{CNV}$ showed increased expression of genes involved in glycolysis, one-carbon metabolism, nucleotide synthesis, TCA cycle, and OXPHOS [31]. Even despite the importance of glycolysis for ECs [63, 116, 117], OXPHOS is required for nucleotide production during in vivo angiogenesis [134, 135]. Several metabolic adaptations were detected in subsets of TECs in NSCLC. To name a few, genes involved in lipid metabolism are upregulated in capillary TECs, while venous TECs exhibit an increased prostaglandin metabolic transcriptome signature [31]. Further, compared to early-stage GGN-ADC, TECs from late-stage SADC are enriched in metabolic processes
[83]. Comparative single-cell analysis of three distinct cancer types (colorectal, lung, ovarian) revealed upregulation of genes involved in glycolysis and OXPHOS in tip cells [84]. Compared to capillary ECs in normal lungs, TECs in lung tumors downregulated the expression of carbonic acid metabolism (characteristic of alveolar ECs), but instead enhanced the expression of genes involved in glycolysis and OXPHOS [84]. Using an EC-tailored genome-scale metabolic model as part of an integrated multi-omics analysis, Aldh18a1 and Sqle were identified as consistently upregulated genes in angiogenic ECs in tumors and CNV and demonstrated to be critical for EC proliferation, migration, and vessel sprouting [31].

\section{Perspectives and therapeutic implications}

Historically, ECs were categorized according to their anatomical position in the vascular tree (e.g., arteries, capillaries, veins) and, more recently, in the vessel sprout (e.g., tip, stalk, phalanx cells). Bulk-omics analyses revealed that ECs exhibited tissue-specific gene signatures. Single-cell analyses, capturing transitory functional states no longer based only on their position in the vascular tree, now reveal that ECs are more plastic and heterogeneous than anticipated. For instance, ECs in a healthy vessel can flexibly adapt to change their transcriptome so that they temporarily differentiate to a new EC phenotype (such as immuno-modulatory ECs), which nonetheless can be identified as a statistically separable phenotype/EC subtype. The evolution to consider these functional EC phenotypes as distinct EC phenotypes is unstoppable, and within the near foreseeable future, these functional EC phenotypes will become targets for new vascular oriented therapies, though it will be challenging to decipher how to target particular EC phenotypes. Given that these EC phenotypes can exist transiently, an option might be to consider strategies to "retune" such functional phenotypes, rather than the "pruning" approach of past/current anti-angiogenic therapy. On the other hand, progress has been made regarding the targeting of different metabolic pathways in ECs to inhibit angiogenesis. Inhibition of PFKFB3 in a pre-clinical model of lung cancer reduced angiogenesis, decreased metastasis, enhanced barrier function, and improved drug delivery [116, 117, 136] (Fig. 4B).

Another major challenge is to identify and to prioritize, among the multiple possible candidates identified by single-cell analyses, the true targets for further clinical translation. Combining single-cell approaches with congruent gene expression analysis across multiple species, tissues, conditions etc. is a powerful strategy to identify biologically relevant targets (Box Novel integrated analysis to identify \& validate scRNA-seq targets). Multi-omics approaches combining spatial and epigenetic information 
with transcriptomic signatures promise to further provide an additional strategy to better address the mechanisms that govern this heterogeneity [104].

\section{Information boxes}

\section{Box 1: Exploitation of EC plasticity for regenerative medicine}

ECs display great plasticity exemplified by their ability to differentiate into numerous EC subtypes and their trans-differentiation into distinct cell lineages, such as hematopoietic stem cells (HSCs) and cardiomyocytes. This plasticity might offer an opportunity to use ECs in clinical applications, in particular in the field of regenerative medicine.

\section{Engineering blood vessels ex vivo}

Ex vivo EC organoid systems are used to study vascular development and neovascularization, to mimic in vivo drug treatments, as well as for cell therapy and transplantation. An established method to create blood vessel organoids is the derivation of ECs from human pluripotent stem cells (hPSCs) in 3D models [137, 138]. ECs can be derived from hPSCs by induction of mesodermal fate and subsequent specification of the vascular fate [139]. One exciting application of hPSC-induced blood vessel organoids is the construction of vascularized tissue grafts [140]. A major challenge in tissue transplantation is the integration of the graft into the recipient's vasculature. Astonishingly, hPSC-derived blood vessel organoids cannot only form fully functional networks in vitro, but also integrate with the host vasculature in vivo, as demonstrated in a model of diabetic vasculopathy, where longterm engraftment was achieved in mice [141].

\section{Cell transfer therapies}

Several pathologies are caused or accompanied by vascular dysfunction and chronic ischemia. Autologous use of ECs in such contexts might offer therapeutic opportunities. For instance, transplantation of CD157+ progenitor ECs can promote endothelial regeneration and new vessel formation [46]. Moreover, as explained above, ECs can be generated from hPSCs for potential cell therapy purposes. The discovery of specialized EC subtypes by single-cell studies also raises the question whether hPSCs-derived ECs can be tuned to phenotypes, which favor angiogenesis. On the other hand, ECs are not only capable of transitioning between different states, but can also transdifferentiate into distinct cell lineages.
Several protocols have been developed to derive different cells from ECs, including HSCs. Here, the mechanistic insights gained from studies investigating blood cell formation from ECs during embryogenesis (a process known as endothelial-to-hematopoietic transition (EHT)) allowed successful transformation of adult ECs from different organs into fully competent HSCs. While the utilization of such EC-derived HSCs in the clinical setting has yet to be tested, these findings hold possible clinical potential for transplantation and cell therapies.

\section{Box 2: Novel integrated analysis to identify \& validate scRNA-seq targets}

Most transcriptomic analyses of ECs differ in study design with respect to species (human vs. mouse) and controls for the pathological setting (healthy ECs from the same organ, healthy ECs from a different organ; and in case of tumors: ECs from peritumoral tissue or ECs from different tumor types). These differences between studies make it challenging to identify common EC subtypes with robust transcriptomic signatures. In addition, prioritization of targets is a formidable challenge when interpreting large datasets. To overcome these challenges, integrated multi-disciplinary approaches (involving scRNA-seq, complemented with proteomics, bulk transcriptomics, and functional studies) or multi-omics approaches (e.g., transcriptomic studies with epi-genomic atlases and/or spatially resolved gene expression studies) are emerging new tools that can facilitate the identification of robust transcriptomic EC phenotypes and/or provide mechanistic insights that govern EC heterogeneity. Here, we provide a brief overview of novel multi-omics and integrated analyses that recently emerged to analyze scRNA transcriptomic data with the goal to identify meaningful targets.

\section{Orthogonal multi-omics and meta-analysis of scRNA-seq data}

This approach was recently used to reconstruct TEC taxonomies in lung cancer, using human (4 different lung tumor subtypes) and mouse (orthotopic model of lung cancer) tissues to identify new tumor-specific EC subtypes not present in healthy tissues. A congruent meta-analysis was performed to identify conserved TEC phenotypes and angiogenic pathways (putative new targets) across these different species (human and mouse) and models, including on top of the above-mentioned datasets, cultured TECs as well as interrogation of publicly available bulk transcriptomics datasets. To further increase the power of target predictions, a meta-analysis of 144 bulk proteomic datasets was performed which, in 
combination with the transcriptomic data, led to the identification of highly conserved (and therefore likely to be functionally relevant) angiogenic targets, among which were genes involved in collagen hydroxylation [6]. Such an integrated approach is becoming increasingly popular and several computational strategies are available to perform such integrated analysis across large datasets [142].

\section{Integration of genome-scale metabolic models (GEM) with scRNA-seq data}

ScRNA-seq studies have been used to profile metabolic gene signatures at the single-cell level [4, 6, 31]. However, transcriptomic studies do not provide insight in metabolic flux activities. GEnome-scale Metabolic models (GEMs) are mathematical computational models of active metabolic reactions, which can be tailored to a particular cell type by integrating multi-omics and other data and can be used to predict the essentiality of particular metabolic reactions. Combining scRNA-seq analyses with the use of an EC-tailored GEM (Endo-GEM) offers the opportunity to prioritize metabolic targets. Indeed, in one study [31], scRNA-seq data were first generated from two models of pathological angiogenesis (choroidal neovascularization (CNV) and tumor angiogenesis) and then integrated with Endo-GEM to identify EC-specific metabolic signatures, congruently upregulated in both models. Using Endo-GEM, 288 essential genes were identified, including genes previously shown to be important for EC metabolism (glycolysis, OXPHOS, fatty acid oxidation, nucleotide synthesis, and salvage), but also genes involved in cholesterol biosynthesis, sphingolipid metabolism, and amino acid metabolism, previously unknown to be essential for vessel formation [31]. Using an integrated approach, new angiogenic target genes (i.e., Sqle and Aldh18al) were identified and functionally validated [31]. Of interest, this integrative approach has been recently applied also to other diseases [143].

\section{Multi-omics platforms}

Emerging multi-omic platforms offer the opportunity to simultaneously profile RNA and DNA and/or protein at the single-cell level and with spatial resolution. Recent examples include scRNA-seq and chromatin accessibility analysis of ECs in a mouse model of disturbed flow [100] as well as the analysis of the chromatin landscape of cardiac ECs [144] and brain ECs [145]. Spatial analyses are also being used to map (endothelial) cell heterogeneity across tissues and conditions including in large cohorts of tissues [146].
Acknowledgements P.C. is supported by Grants from Methusalem funding (Flemish government), the Fund for Scientific ResearchFlanders (FWO-Vlaanderen), the European Research Council (ERC Proof of concept ERC-713758; ERC Advanced Research Grant EUERC743074), and a NNF Laureate Research Grant from Novo Nordisk Foundation (Denmark); L.M.B. is supported by a Marie-SklodowskaCurie fellowship; A.C. is supported by a 'Fonds voor Wetenschappelijk Onderzoek' (FWO) doctoral fellowship. Figures were generated with Biorender.com.

Open Access This article is licensed under a Creative Commons Attribution 4.0 International License, which permits use, sharing, adaptation, distribution and reproduction in any medium or format, as long as you give appropriate credit to the original author(s) and the source, provide a link to the Creative Commons licence, and indicate if changes were made. The images or other third party material in this article are included in the article's Creative Commons licence, unless indicated otherwise in a credit line to the material. If material is not included in the article's Creative Commons licence and your intended use is not permitted by statutory regulation or exceeds the permitted use, you will need to obtain permission directly from the copyright holder. To view a copy of this licence, visit http://creativecommons.org/licenses/by/4.0/.

\section{References}

1. Aird WC (2007) Phenotypic heterogeneity of the endothelium: I. structure, function, and mechanisms. Circ Res 100:158-173. https://doi.org/10.1161/01.RES.0000255691.76142.4a

2. Potente M, Makinen T (2017) Vascular heterogeneity and specialization in development and disease. Nat Rev Mol Cell Biol 18:477-494. https://doi.org/10.1038/nrm.2017.36

3. Aird WC (2012) Endothelial cell heterogeneity. Cold Spring Harb Perspect Med 2:a006429. https://doi.org/10.1101/cshpe rspect.a006429

4. Kalucka J et al (2020) Single-cell transcriptome atlas of murine endothelial cells. Cell 180(4):764-779. https://doi.org/10.1016/j. cell.2020.01.015

5. Jakab M, Augustin HG (2020) Understanding angiodiversity: insights from single cell biology. Development. https://doi.org/ $10.1242 /$ dev. 146621

6. Goveia J et al (2020) An integrated gene expression landscape profiling approach to identify lung tumor endothelial cell heterogeneity and angiogenic candidates. Cancer Cell 37:421. https:// doi.org/10.1016/j.ccell.2020.03.002

7. Chavkin NW, Hirschi KK (2020) Single cell analysis in vascular biology. Front Cardiovasc Med 7:42. https://doi.org/10.3389/ fcvm.2020.00042

8. Paik DT et al (2020) Single-cell RNA sequencing unveils unique transcriptomic signatures of organ-specific endothelial cells. Circulation 142:1848-1862. https://doi.org/10.1161/CIRCULATIO NAHA.119.041433

9. Dumas SJ et al (2020) Single-cell RNA sequencing reveals renal endothelium heterogeneity and metabolic adaptation to water deprivation. J Am Soc Nephrol 31:118-138. https://doi.org/10. 1681/ASN.2019080832

10. Vanlandewijck M et al (2018) A molecular atlas of cell types and zonation in the brain vasculature. Nature 554:475-480. https:// doi.org/10.1038/nature25739

11. Koch PS, Lee KH, Goerdt S, Augustin HG (2021) Angiodiversity and organotypic functions of sinusoidal endothelial cells. Angiogenesis. https://doi.org/10.1007/s10456-021-09780-y 
12. Su T et al (2018) Single-cell analysis of early progenitor cells that build coronary arteries. Nature 559:356-362. https://doi.org/10. 1038/s41586-018-0288-7

13. Augustin HG, Koh GY (2017) Organotypic vasculature: from descriptive heterogeneity to functional pathophysiology. Science. https://doi.org/10.1126/science.aal2379

14. Dejana E, Hirschi KK, Simons M (2017) The molecular basis of endothelial cell plasticity. Nat Commun 8:14361. https://doi.org/ 10.1038/ncomms 14361

15. Ma W, Oliver G (2017) Lymphatic endothelial cell plasticity in development and disease. Physiology 32:444-452. https://doi. org/10.1152/physiol.00015.2017

16. Greenspan LJ, Weinstein BM (2021) To be or not to be: endothelial cell plasticity in development, repair, and disease. Angiogenesis. https://doi.org/10.1007/s10456-020-09761-7

17. Platel V, Faure S, Corre I, Clere N (2019) Endothelial-to-mesenchymal transition (EndoMT): roles in tumorigenesis, metastatic extravasation and therapy resistance. J Oncol 2019:8361945. https://doi.org/10.1155/2019/8361945

18. Oliver G, Srinivasan RS (2010) Endothelial cell plasticity: how to become and remain a lymphatic endothelial cell. Development 137:363-372. https://doi.org/10.1242/dev.035360

19. Dumas SJ, Garcia-Caballero M, Carmeliet P (2020) Metabolic signatures of distinct endothelial phenotypes. Trends Endocrinol Metab 31:580-595. https://doi.org/10.1016/j.tem.2020.05.009

20. Paik DT, Cho S, Tian L, Chang HY, Wu JC (2020) Singlecell RNA sequencing in cardiovascular development, disease and medicine. Nat Rev Cardiol 17:457-473. https://doi.org/10. 1038/s41569-020-0359-y

21. Rajendran $P$ et al (2013) The vascular endothelium and human diseases. Int J Biol Sci 9:1057-1069. https://doi.org/10.7150/ ijbs. 7502

22. Carmeliet P, Jain RK (2011) Principles and mechanisms of vessel normalization for cancer and other angiogenic diseases. Nat Rev Drug Discov 10:417-427. https://doi.org/10.1038/nrd34 55

23. You LR et al (2005) Suppression of Notch signalling by the COUP-TFII transcription factor regulates vein identity. Nature 435:98-104. https://doi.org/10.1038/nature03511

24. Red-Horse K, Ueno H, Weissman IL, Krasnow MA (2010) Coronary arteries form by developmental reprogramming of venous cells. Nature 464:549-553. https://doi.org/10.1038/nature08873

25. Conway EM, Collen D, Carmeliet P (2001) Molecular mechanisms of blood vessel growth. Cardiovasc Res 49:507-521. https://doi.org/10.1016/s0008-6363(00)00281-9

26. Nicenboim $\mathbf{J}$ et al (2015) Lymphatic vessels arise from specialized angioblasts within a venous niche. Nature 522:56-61. https://doi.org/10.1038/nature14425

27. Mack JJ, Iruela-Arispe ML (2018) NOTCH regulation of the endothelial cell phenotype. Curr Opin Hematol 25:212-218. https://doi.org/10.1097/MOH.0000000000000425

28. Luo W et al (2020) Arterialization requires the timely suppression of cell growth. Nature. https://doi.org/10.1038/ s41586-020-3018-x

29. Khan S et al (2019) EndoDB: a database of endothelial cell transcriptomics data. Nucleic Acids Res 47:D736-D744. https://doi. org/10.1093/nar/gky997

30. Franzen O, Gan LM, Bjorkegren JLM (2019) PanglaoDB: a web server for exploration of mouse and human single-cell RNA sequencing data. Database. https://doi.org/10.1093/database/ baz046

31. Rohlenova K et al (2020) Single-cell RNA sequencing maps endothelial metabolic plasticity in pathological angiogenesis. Cell Metab 31:862-877. https://doi.org/10.1016/j.cmet.2020. 03.009
32. Nolan DJ et al (2013) Molecular signatures of tissue-specific microvascular endothelial cell heterogeneity in organ maintenance and regeneration. Dev Cell 26:204-219. https://doi.org/ 10.1016/j.devcel.2013.06.017

33. VanHorn S, Morris SA (2020) Next-generation lineage tracing and fate mapping to interrogate development. Dev Cell. https:// doi.org/10.1016/j.devcel.2020.10.021

34. Bowling S et al (2020) An Engineered CRISPR-Cas9 Mouse Line for Simultaneous Readout of Lineage Histories and Gene Expression Profiles in Single Cells. Cell 181:1410-1422. https:// doi.org/10.1016/j.cell.2020.04.048

35. Weijts B et al (2018) Blood flow-induced Notch activation and endothelial migration enable vascular remodeling in zebrafish embryos. Nat Commun 9:5314. https://doi.org/10.1038/ s41467-018-07732-7

36. le Noble F et al (2005) Control of arterial branching morphogenesis in embryogenesis: go with the flow. Cardiovasc Res 65:619-628. https://doi.org/10.1016/j.cardiores.2004.09.018

37. Chen Q et al (2016) Endothelial cells are progenitors of cardiac pericytes and vascular smooth muscle cells. Nat Commun 7:12422. https://doi.org/10.1038/ncomms 12422

38. Lis R et al (2017) Conversion of adult endothelium to immunocompetent haematopoietic stem cells. Nature 545:439-445. https://doi.org/10.1038/nature22326

39. Canu G et al (2020) Analysis of endothelial-to-haematopoietic transition at the single cell level identifies cell cycle regulation as a driver of differentiation. Genome Biol 21:157. https://doi. org/10.1186/s13059-020-02058-4

40. Chestnut B, Casie Chetty S, Koenig AL, Sumanas S (2020) Single-cell transcriptomic analysis identifies the conversion of zebrafish Etv2-deficient vascular progenitors into skeletal muscle. Nat Commun 11:2796. https://doi.org/10.1038/ s41467-020-16515-y

41. De Val S et al (2008) Combinatorial regulation of endothelial gene expression by ets and forkhead transcription factors. Cell 135:1053-1064. https://doi.org/10.1016/j.cell.2008.10.049

42. Sumanas S, Choi K (2016) ETS transcription factor ETV2/ER71/ Etsrp in hematopoietic and vascular development. Curr Top Dev Biol 118:77-111. https://doi.org/10.1016/bs.ctdb.2016.01.005

43. Kardon G, Campbell JK, Tabin CJ (2002) Local extrinsic signals determine muscle and endothelial cell fate and patterning in the vertebrate limb. Dev Cell 3:533-545. https://doi.org/10.1016/ s1534-5807(02)00291-5

44. Mayeuf-Louchart A et al (2014) Notch regulation of myogenic versus endothelial fates of cells that migrate from the somite to the limb. Proc Natl Acad Sci 111:8844-8849. https://doi.org/10. 1073/pnas. 1407606111

45. Medici D et al (2010) Conversion of vascular endothelial cells into multipotent stem-like cells. Nat Med 16:1400-1406. https:// doi.org/10.1038/nm.2252

46. Qiu J, Hirschi KK (2019) Endothelial cell development and its application to regenerative medicine. Circ Res 125:489-501. https://doi.org/10.1161/CIRCRESAHA.119.311405

47. Ng AHM et al (2020) A comprehensive library of human transcription factors for cell fate engineering. Nat Biotechnol. https:// doi.org/10.1038/s41587-020-0742-6

48. Augustin HG (2001) Tubes, branches, and pillars: the many ways of forming a new vasculature. Circ Res 89:645-647

49. De Spiegelaere $\mathrm{W}$ et al (2012) Intussusceptive angiogenesis: a biologically relevant form of angiogenesis. J Vasc Res 49:390 404. https://doi.org/10.1159/000338278

50. Fan X et al (2019) Single-cell reconstruction of follicular remodeling in the human adult ovary. Nat Commun 10:3164. https:// doi.org/10.1038/s41467-019-11036-9 
51. Wang W et al (2020) Single-cell transcriptomic atlas of the human endometrium during the menstrual cycle. Nat Med 26:1644-1653. https://doi.org/10.1038/s41591-020-1040-z

52. Potente M, Gerhardt H, Carmeliet P (2011) Basic and therapeutic aspects of angiogenesis. Cell 146:873-887. https://doi.org/10. 1016/j.cell.2011.08.039

53. Hellstrom $\mathrm{M}$ et al (2007) Dll4 signalling through Notch1 regulates formation of tip cells during angiogenesis. Nature 445:776780. https://doi.org/10.1038/nature05571

54. Hellstrom M, Phng LK, Gerhardt H (2007) VEGF and Notch signaling: the yin and yang of angiogenic sprouting. Cell Adhes Migr 1:133-136. https://doi.org/10.4161/cam.1.3.4978

55. Jakobsson L et al (2010) Endothelial cells dynamically compete for the tip cell position during angiogenic sprouting. Nat Cell Biol 12:943-953. https://doi.org/10.1038/ncb2103

56. Tammela T et al (2011) VEGFR-3 controls tip to stalk conversion at vessel fusion sites by reinforcing Notch signalling. Nat Cell Biol 13:1202-1213. https://doi.org/10.1038/ncb2331

57. Boareto M, Jolly MK, Ben-Jacob E, Onuchic JN (2015) Jagged mediates differences in normal and tumor angiogenesis by affecting tip-stalk fate decision. Proc Natl Acad Sci 112:E3836-3844. https://doi.org/10.1073/pnas.1511814112

58. McDonald AI et al (2018) Endothelial regeneration of large vessels is a biphasic process driven by local cells with distinct proliferative capacities. Cell Stem Cell 23:210-225. https://doi.org/ 10.1016/j.stem.2018.07.011

59. Wang $\mathrm{Z}$ et al (2020) Cell-type-specific gene regulatory networks underlying murine neonatal heart regeneration at single-cell resolution. Cell Rep 33:108472. https://doi.org/10.1016/j.celrep. 2020.108472

60. Blanco R, Gerhardt H (2013) VEGF and Notch in tip and stalk cell selection. Cold Spring Harb Perspect Med 3:a006569. https://doi.org/10.1101/cshperspect.a006569

61. Chen W et al (2019) The endothelial tip-stalk cell selection and shuffling during angiogenesis. J Cell Commun Signal 13:291301. https://doi.org/10.1007/s12079-019-00511-z

62. Herbert SP et al (2009) Arterial-venous segregation by selective cell sprouting: an alternative mode of blood vessel formation. Science 326:294-298. https://doi.org/10.1126/science.1178577

63. Li X, Sun X, Carmeliet P (2019) Hallmarks of endothelial cell metabolism in health and disease. Cell Metab 30:414-433. https://doi.org/10.1016/j.cmet.2019.08.011

64. Bergmann $\mathrm{O}$ et al (2015) Dynamics of cell generation and turnover in the human heart. Cell 161:1566-1575. https://doi.org/10. 1016/j.cell.2015.05.026

65. Wakabayashi T et al (2018) CD157 marks tissue-resident endothelial stem cells with homeostatic and regenerative properties. Cell Stem Cell 22:384-397.e386. https://doi.org/10.1016/j. stem.2018.01.010

66. Lukowski SW et al (2019) Single-cell transcriptional profiling of aortic endothelium identifies a hierarchy from endovascular progenitors to differentiated cells. Cell Rep 27:2748-2758. https:// doi.org/10.1016/j.celrep.2019.04.102

67. Gillich A et al (2020) Capillary cell-type specialization in the alveolus. Nature. https://doi.org/10.1038/s41586-020-2822-7

68. Brulois K et al (2020) A molecular map of murine lymph node blood vascular endothelium at single cell resolution. Nat Commun 11:3798. https://doi.org/10.1038/s41467-020-17291-5

69. Deng J et al (2020) Single-cell gene profiling and lineage tracing analyses revealed novel mechanisms of endothelial repair by progenitors. Cell Mol Life Sci 77:5299-5320. https://doi.org/10. 1007/s00018-020-03480-4

70. Chen $\mathrm{Q}$ et al (2018) Genetic lineage tracing analysis of c-kit(+) stem/progenitor cells revealed a contribution to vascular injuryinduced neointimal lesions. J Mol Cell Cardiol 121:277-286. https://doi.org/10.1016/j.yjmcc.2018.07.252
71. Ingram DA et al (2005) Vessel wall-derived endothelial cells rapidly proliferate because they contain a complete hierarchy of endothelial progenitor cells. Blood 105:2783-2786. https://doi. org/10.1182/blood-2004-08-3057

72. Naito H, Kidoya H, Sakimoto S, Wakabayashi T, Takakura N (2012) Identification and characterization of a resident vascular stem/progenitor cell population in preexisting blood vessels. EMBO J 31:842-855. https://doi.org/10.1038/emboj.2011.465

73. Khacho M, Slack RS (2017) Mitochondrial activity in the regulation of stem cell self-renewal and differentiation. Curr Opin Cell Biol 49:1-8. https://doi.org/10.1016/j.ceb.2017.11.003

74. Zhang $\mathrm{H}$ et al (2016) $\mathrm{NAD}(+)$ repletion improves mitochondrial and stem cell function and enhances life span in mice. Science 352:1436-1443. https://doi.org/10.1126/science.aaf2693

75. Vila Ellis L et al (2020) Epithelial vegfa specifies a distinct endothelial population in the mouse lung. Dev Cell 52:617-630. https://doi.org/10.1016/j.devcel.2020.01.009

76. St Croix B et al (2000) Genes expressed in human tumor endothelium. Science 289:1197-1202. https://doi.org/10.1126/science. 289.5482.1197

77. Viallard C, Larrivee B (2017) Tumor angiogenesis and vascular normalization: alternative therapeutic targets. Angiogenesis 20:409-426. https://doi.org/10.1007/s10456-017-9562-9

78. Seaman S et al (2007) Genes that distinguish physiological and pathological angiogenesis. Cancer Cell 11:539-554. https://doi. org/10.1016/j.ccr.2007.04.017

79. Luo W et al (2013) Isolation and genome-wide expression and methylation characterization of CD31+ cells from normal and malignant human prostate tissue. Oncotarget 4:1472-1483. https://doi.org/10.18632/oncotarget.1269

80. Sun $\mathrm{Z}$ et al (2018) Single-cell RNA sequencing reveals gene expression signatures of breast cancer-associated endothelial cells. Oncotarget 9:10945-10961. https://doi.org/10.18632/ oncotarget. 23760

81. Lambrechts D et al (2018) Phenotype molding of stromal cells in the lung tumor microenvironment. Nat Med 24:1277-1289. https://doi.org/10.1038/s41591-018-0096-5

82. Baudino TA et al (2002) c-Myc is essential for vasculogenesis and angiogenesis during development and tumor progression. Genes Dev 16:2530-2543. https://doi.org/10.1101/gad.10246 02

83. Lu T et al (2020) Single-cell transcriptome atlas of lung adenocarcinoma featured with ground glass nodules. Cell Discov 6:69. https://doi.org/10.1038/s41421-020-00200-x

84. Qian J et al (2020) A pan-cancer blueprint of the heterogeneous tumor microenvironment revealed by single-cell profiling. Cell Res 30:745-762. https://doi.org/10.1038/s41422-020-0355-0

85. Ribatti D, Djonov V (2012) Intussusceptive microvascular growth in tumors. Cancer Lett 316:126-131. https://doi.org/10. 1016/j.canlet.2011.10.040

86. Diaz-Flores L et al (2020) Participation of intussusceptive angiogenesis in the morphogenesis of lobular capillary hemangioma. Sci Rep 10:4987. https://doi.org/10.1038/s41598-020-61921-3

87. Kuczynski EA, Vermeulen PB, Pezzella F, Kerbel RS, Reynolds AR (2019) Vessel co-option in cancer. Nat Rev Clin Oncol 16:469-493. https://doi.org/10.1038/s41571-019-0181-9

88. Hlushchuk R et al (2008) Tumor recovery by angiogenic switch from sprouting to intussusceptive angiogenesis after treatment with PTK787/ZK222584 or ionizing radiation. Am J Pathol 173:1173-1185. https://doi.org/10.2353/ajpath.2008.071131

89. Hlushchuk R, Makanya AN, Djonov V (2011) Escape mechanisms after antiangiogenic treatment, or why are the tumors growing again? Int J Dev Biol 55:563-567. https://doi.org/10. 1387/ijdb.103231rh 
90. Wadman M, Couzin-Frankel J, Kaiser J, Matacic C (2020) A rampage through the body. Science 368:356-360. https://doi.org/ 10.1126/science.368.6489.356

91. Teuwen LA, Geldhof V, Pasut A, Carmeliet P (2020) COVID-19: the vasculature unleashed. Nat Rev Immunol 20:389-391. https:// doi.org/10.1038/s41577-020-0343-0

92. Ackermann $\mathrm{M}$ et al (2020) Pulmonary vascular endothelialitis, thrombosis, and angiogenesis in Covid-19. N Engl J Med 383:120-128. https://doi.org/10.1056/NEJMoa2015432

93. Ackermann M, Mentzer SJ, Kolb M, Jonigk D (2020) Inflammation and intussusceptive angiogenesis in COVID-19: everything in and out of flow. Eur Respir J. https://doi.org/10.1183/13993 003.03147-2020

94. Price LC, McCabe C, Garfield B, Wort SJ (2020) Thrombosis and COVID-19 pneumonia: the clot thickens! Eur Respir J. https://doi.org/10.1183/13993003.01608-2020

95. Rovas A et al (2020) Microvascular dysfunction in COVID19: the MYSTIC study. Angiogenesis. https://doi.org/10.1007/ s10456-020-09753-7

96. Minamino T, Komuro I (2006) Regeneration of the endothelium as a novel therapeutic strategy for acute lung injury. J Clin Invest 116:2316-2319. https://doi.org/10.1172/JCI29637

97. Cosgrove GP et al (2004) Pigment epithelium-derived factor in idiopathic pulmonary fibrosis: a role in aberrant angiogenesis. Am J Respir Crit Care Med 170:242-251. https://doi.org/10. 1164/rccm.200308-1151OC

98. Gola A et al (2020) Commensal-driven immune zonation of the liver promotes host defence. Nature. https://doi.org/10.1038/ s41586-020-2977-2

99. Jambusaria A et al (2020) Endothelial heterogeneity across distinct vascular beds during homeostasis and inflammation. Elife. https://doi.org/10.7554/eLife.51413

100. Andueza A et al (2020) Endothelial reprogramming by disturbed flow revealed by single-cell RNA and chromatin accessibility study. Cell Rep 33:108491. https://doi.org/10.1016/j.celrep.2020. 108491

101. Zhao Q et al (2020) Heterogeneity and chimerism of endothelial cells revealed by single-cell transcriptome in orthotopic liver tumors. Angiogenesis 23:581-597. https://doi.org/10.1007/ s10456-020-09727-9

102. Pober JS, Merola J, Liu R, Manes TD (2017) Antigen presentation by vascular cells. Front Immunol 8:1907. https://doi.org/10. 3389/fimmu.2017.01907

103. Choi SY, Gout PW, Collins CC, Wang Y (2012) Epithelial immune cell-like transition (EIT): a proposed transdifferentiation process underlying immune-suppressive activity of epithelial cancers. Differentiation 83:293-298. https://doi.org/10.1016/j. diff.2012.02.005

104. Cusanovich DA et al (2018) A single-cell atlas of in vivo mammalian chromatin accessibility. Cell 174:1309-1324 e1318. https://doi.org/10.1016/j.cell.2018.06.052

105. Stahl PL et al (2016) Visualization and analysis of gene expression in tissue sections by spatial transcriptomics. Science 353:78-82. https://doi.org/10.1126/science.aaf2403

106. Krausgruber T et al (2020) Structural cells are key regulators of organ-specific immune responses. Nature 583:296-302. https:// doi.org/10.1038/s41586-020-2424-4

107. Cai Q, Moore SA, Hendricks AR, Torrealba JR (2020) Upregulation of endothelial HLA class II is a marker of antibody-mediated rejection in heart allograft biopsies. Transplant Proc 52:11921197. https://doi.org/10.1016/j.transproceed.2020.01.049

108. Al-Lamki RS, Bradley JR, Pober JS (2008) Endothelial cells in allograft rejection. Transplantation 86:1340-1348. https://doi. org/10.1097/TP.0b013e3181891d8b
109. Manavski Y et al (2018) Clonal expansion of endothelial cells contributes to ischemia-induced neovascularization. Circ Res 122:670-677. https://doi.org/10.1161/CIRCRESAHA.117. 312310

110. Li Z et al (2019) Single-cell transcriptome analyses reveal novel targets modulating cardiac neovascularization by resident endothelial cells following myocardial infarction. Eur Heart $\mathbf{J}$ 40:2507-2520. https://doi.org/10.1093/eurheartj/ehz305

111. Ackermann M et al (2020) Morphomolecular motifs of pulmonary neoangiogenesis in interstitial lung diseases. Eur Respir J. https://doi.org/10.1183/13993003.00933-2019

112. Notoya M, Shinosaki T, Kobayashi T, Sakai T, Kurihara H (2003) Intussusceptive capillary growth is required for glomerular repair in rat Thy-1.1 nephritis. Kidney Int 63:1365-1373. https://doi. org/10.1046/j.1523-1755.2003.00876.x

113. Konerding MA et al (2010) Inflammation-induced intussusceptive angiogenesis in murine colitis. Anat Rec 293:849-857. https://doi.org/10.1002/ar.21110

114. Adams TS et al (2020) Single-cell RNA-seq reveals ectopic and aberrant lung-resident cell populations in idiopathic pulmonary fibrosis. Sci Adv. https://doi.org/10.1126/sciadv.aba1983

115. De Bock K, Georgiadou M, Carmeliet P (2013) Role of endothelial cell metabolism in vessel sprouting. Cell Metab 18:634-647. https://doi.org/10.1016/j.cmet.2013.08.001

116. De Bock K et al (2013) Role of PFKFB3-driven glycolysis in vessel sprouting. Cell 154:651-663. https://doi.org/10.1016/j. cell.2013.06.037

117. Schoors S et al (2014) Partial and transient reduction of glycolysis by PFKFB3 blockade reduces pathological angiogenesis. Cell Metab 19:37-48. https://doi.org/10.1016/j.cmet.2013.11.008

118. Schoors $\mathrm{S}$ et al (2015) Fatty acid carbon is essential for dNTP synthesis in endothelial cells. Nature 520:192-197. https://doi. org/10.1038/nature 14362

119. Kalucka J et al (2018) Quiescent Endothelial Cells Upregulate Fatty Acid beta-Oxidation for Vasculoprotection via Redox Homeostasis. Cell Metab 28:881-894. https://doi.org/10.1016/j. cmet.2018.07.016

120. Wong BW et al (2017) The role of fatty acid beta-oxidation in lymphangiogenesis. Nature 542:49-54. https://doi.org/10.1038/ nature 21028

121. Eelen $\mathrm{G}$ et al (2018) Endothelial cell metabolism. Physiol Rev 98:3-58. https://doi.org/10.1152/physrev.00001.2017

122. Huang $\mathrm{H}$ et al (2017) Role of glutamine and interlinked asparagine metabolism in vessel formation. EMBO J 36:2334-2352. https://doi.org/10.15252/embj.201695518

123. Falkenberg KD, Rohlenova K, Luo Y, Carmeliet P (2019) The metabolic engine of endothelial cells. Nat Metab 1:937-946. https://doi.org/10.1038/s42255-019-0117-9

124. Garcia-Caballero $M$ et al (2019) Role and therapeutic potential of dietary ketone bodies in lymph vessel growth. Nat Metab 1:666-675. https://doi.org/10.1038/s42255-019-0087-y

125. Sun $\mathrm{S}$ et al (2015) Constitutive activation of mTORC1 in endothelial cells leads to the development and progression of lymphangiosarcoma through VEGF autocrine signaling. Cancer Cell 28:758-772. https://doi.org/10.1016/j.ccell.2015.10.004

126. Wilhelm K et al (2016) FOXO1 couples metabolic activity and growth state in the vascular endothelium. Nature 529:216-220. https://doi.org/10.1038/nature16498

127. Doddaballapur A et al (2015) Laminar shear stress inhibits endothelial cell metabolism via KLF2-mediated repression of PFKFB3. Arterioscler Thromb Vasc Biol 35:137-145. https:// doi.org/10.1161/ATVBAHA.114.304277

128. Bruning $U$ et al (2018) Impairment of angiogenesis by fatty acid synthase inhibition involves mTOR malonylation. Cell Metab 28:866-880. https://doi.org/10.1016/j.cmet.2018.07.019 
129. Stone OA et al (2018) Loss of pyruvate kinase M2 limits growth and triggers innate immune signaling in endothelial cells. Nat Commun 9:4077. https://doi.org/10.1038/s41467-018-06406-8

130. Kim B et al (2018) Endothelial pyruvate kinase M2 maintains vascular integrity. J Clin Invest 128:4543-4556. https://doi.org/ 10.1172/JCI120912

131. Teuwen LA, Geldhof V, Carmeliet P (2019) How glucose, glutamine and fatty acid metabolism shape blood and lymph vessel development. Dev Biol 447:90-102. https://doi.org/10.1016/j. ydbio.2017.12.001

132. Son NH et al (2018) Endothelial cell CD36 optimizes tissue fatty acid uptake. J Clin Invest 128:4329-4342. https://doi.org/ 10.1172/JCI99315

133. He L et al (2018) Single-cell RNA sequencing of mouse brain and lung vascular and vessel-associated cell types. Sci Data 5:180160. https://doi.org/10.1038/sdata.2018.160

134. Schiffmann LM et al (2020) Mitochondrial respiration controls neoangiogenesis during wound healing and tumour growth. Nat Commun 11:3653. https://doi.org/10.1038/s41467-020-17472-2

135. Diebold LP et al (2019) Mitochondrial complex III is necessary for endothelial cell proliferation during angiogenesis. Nat Metab 1:158-171. https://doi.org/10.1038/s42255-018-0011-x

136. Cantelmo AR et al (2016) Inhibition of the glycolytic activator PFKFB3 in endothelium induces tumor vessel normalization, impairs metastasis, and improves chemotherapy. Cancer Cell 30:968-985. https://doi.org/10.1016/j.ccell.2016.10.006

137. Samuel R, Duda DG, Fukumura D, Jain RK (2015) Vascular diseases await translation of blood vessels engineered from stem cells. Sci Transl Med 7:309rv6-306. https://doi.org/10.1126/scitr anslmed.aaa1805

138. Cheung C, Bernardo AS, Trotter MW, Pedersen RA, Sinha S (2012) Generation of human vascular smooth muscle subtypes provides insight into embryological origin-dependent disease susceptibility. Nat Biotechnol 30:165-173. https://doi.org/10. $1038 /$ nbt.2107
139. Lian $X$ et al (2014) Efficient differentiation of human pluripotent stem cells to endothelial progenitors via small-molecule activation of WNT signaling. Stem Cell Rep 3:804-816. https://doi. org/10.1016/j.stemcr.2014.09.005

140. Mao AS, Mooney DJ (2015) Regenerative medicine: current therapies and future directions. Proc Natl Acad Sci U S A 112:14452-14459. https://doi.org/10.1073/pnas.1508520112

141. Wimmer RA et al (2019) Human blood vessel organoids as a model of diabetic vasculopathy. Nature 565:505-510. https://doi. org/10.1038/s41586-018-0858-8

142. Shafer MER (2019) Cross-species analysis of single-cell transcriptomic data. Front Cell Dev Biol 7:175. https://doi.org/10. 3389/fcell.2019.00175

143. Granata I, Troiano E, Sangiovanni M, Guarracino MR (2019) Integration of transcriptomic data in a genome-scale metabolic model to investigate the link between obesity and breast cancer. BMC Bioinform 20:162. https://doi.org/10.1186/ s12859-019-2685-9

144. Yucel $\mathrm{N}$ et al (2020) Cardiac endothelial cells maintain open chromatin and expression of cardiomyocyte myofibrillar genes. Elife. https://doi.org/10.7554/eLife.55730

145. Sabbagh MF et al (2018) Transcriptional and epigenomic landscapes of CNS and non-CNS vascular endothelial cells. Elife. https://doi.org/10.7554/eLife.36187

146. Delorey TM et al (2021) A single-cell and spatial atlas of autopsy tissues reveals pathology and cellular targets of SARS-CoV-2. bioRxiv. https://doi.org/10.1101/2021.02.25.430130

Publisher's Note Springer Nature remains neutral with regard to jurisdictional claims in published maps and institutional affiliations. 